\section{Potenciais usos dos AP-DRG para discriminar - perfil da assistência de unidades hospitalares}

\author{
Potential uses of AP-DRG to describe \\ the health care profile in hospital units
}

\author{
Marina Ferreira de Noronha 1 \\ Margareth Crisóstomo Portela 1 \\ Maria Lúcia Lebrão 2
}

\section{Abstract}

1 Escola Nacional de Saúde Pública, Fundação Oswaldo Cruz, Rio de Janeiro, Brasil.

2 Faculdade de Saúde Pública, Universidade de São Paulo,

São Paulo, Brasil.

Correspondência M. F. Noronha Escola Nacional de Saúde Pública, Fundação Oswaldo Cruz. Av. Leopoldo Bulhões 1480, Rio de Janeiro, $R J$ 21041-210, Brasil. marinan@ensp.fiocruz.br
The All Patient Diagnosis Related Groups (AP$D R G$ ) provide a classification system for general hospital inpatients, aggregating hospitalizations based on resource use and clinical criteria. The different versions of the AP-DRG have been applied to inpatient care management and reimbursement. This paper aimed to describe the classification and explore the potential for generating information on inpatient care management based on data from the Ribeirão Preto region (São Paulo State, Brazil) in 1997, including public, private, and charity hospitals. We compared average length of stay related to DRGs in the Ribeirão Preto region and the United States. Using the relative cost weights of the AP-DRGs constructed for New York State, we verified the profile of inpatient care provided by 30 hospitals in the Ribeirão Preto region, and reimbursement for hospital care provided to patients referred from other municipalities and covered by the Unified National Health System (SUS). Our findings indicate the applicability of the classification to inform the decision-making process on inpatient care regionalization and organization in levels of complexity, as well as for improvement of inpatient care monitoring and reimbursement.

Health Service; Health Resources; Residence Time; Quality Management

\section{Introdução}

Os Diagnosis Related Groups (DRG) constituem-se em um sistema de classificação de pacientes construído com o intuito de instrumentalizar a gestão hospitalar, viabilizando a mensuração e avaliação do desempenho dos hospitais. O sistema tem como foco a definição da produção final do hospital, caracterizada como o conjunto de bens e serviços prestados aos pacientes para tratar problemas de saúde específicos 1 . Portanto, na construção da classificação buscou-se relacionar os tipos de pacientes atendidos pelo hospital, ou seja, o seu perfil nosológico, ao conjunto de bens e serviços consumidos durante sua hospitalização, resultando no agrupamento de pacientes com perfis clínicos e de consumo de recursos semelhantes. O tempo de permanência hospitalar foi utilizado como variável de referência do consumo de recursos e, posteriormente, a classificação foi validada utilizando-se informações sobre os custos. Ela é específica para internações em hospitais gerais, de curta permanência, denominados hospitais de agudos 2,3.

Essa classificação, desenvolvida na Universidade de Yale, nos Estados Unidos, no final da década de 60 e ao longo da década de 70, teve o objetivo de definir o produto hospitalar para fins de monitoramento da utilização de serviços, bem como de avaliação e gerência de qualidade da atenção hospitalar. Na década de 70, 
a classificação foi aplicada no Estado de Nova Jersey e, na década de 80 , foi revisada e aprimorada para ser utilizada, em 1983, como unidade de referência para o reembolso financeiro aos hospitais credenciados pelo governo americano, no atendimento a pacientes do seguro de saúde Medicare 1,3,4,5.

A característica mais importante dos DRGs é permitir a comparação do desempenho hospitalar. Essa qualidade da classificação, associada à facilidade na obtenção dos dados de hospitalização necessários, encontrados em resumos de saída hospitalares, é o que permite sua utilização para diversos propósitos dentro da grande área de gestão dos serviços hospitalares. Sua aplicação tem sido voltada para o pagamento de hospitalizações e para o planejamento e gestão do sistema de atenção hospitalar em seus diversos níveis 6 .

Essa classificação vem sendo aprimorada, modificada, testada e utilizada em diversos países do mundo desde o final da década de 80 . Desse modo, diversas versões e novas classificações de DRG vêm sendo disponibilizadas resultantes: da incorporação de críticas feitas às versões anteriores, da identificação de novas necessidades, do aprimoramento das informações da atenção hospitalar e do desenvolvimento de novas tecnologias. Alguns exemplos dessas novas classificações são: o DRG do Medicare (DRG), o All Patient DRGs (AP-DRG), o All Patient Refined DRG (APR-DRG) $e$ o International Refined DRG (IR-DRG). Todos os exemplos citados acima passam por revisão anual ou bianual, e os aprimoramentos realizados em uma versão tem sido, geralmente, incorporados às outras versões das demais classificações. O DRG do Medicare é mais adequado para discriminar a atenção hospitalar de idosos por ser esse o seu uso nos Estados Unidos. A classificação AP-DRG é adequada para descrever o atendimento a todas as faixas etárias. A APR-DRG é um aprimoramento da AP-DRG e tem duas vertentes: uma voltada para o consumo de recursos e a outra para o risco de morte. A IR-DRG tem como referência as duas classificações anteriores e foi construída tendo como base hospitalizações em países da Europa, permitindo a utilização de diversas classificações de diagnósticos além da utilizada nos Estados Unidos. A segunda versão da IR-DRG, ainda em fase de testes, permite, também, a discriminação da atenção ambulatorial prestada dentro dos hospitais 3,7,8,9.

Em paralelo ao desenvolvimento dos DRGs, e também em concorrência a eles, numerosas alternativas de classificações de pacientes para mensurar o case-mix têm sido propostas nos últimos vinte anos. Cada uma descreve um aspecto particular do case-mix hospitalar, ambulatorial, de hospitais especializados, dentre outros, buscando inter-relacionar atributos distintos de pacientes, como a gravidade da doença, prognóstico, dificuldade no tratamento, necessidade de intervenção e intensidade de recursos 2,10,11,12,13,14,15,16,17,18,19,20. O termo case-mix foi definido por Fetter et al. 2 como a proporção relativa de diferentes tipos de pacientes que o hospital trata.

No Brasil, as três esferas de governo vêm realizando o reembolso financeiro dos serviços de atenção hospitalar, tanto os públicos como os realizados por hospitais privados contratados pelo SUS, utilizando, como unidade de pagamento, procedimentos que compõem a classificação de procedimentos hospitalares, elaborada no começo da década de 8021 . A classificação parte de uma lista de procedimentos médicos que são classificados em cirúrgicos e clínicos e organizados em subgrupos baseados, principalmente, em sistemas orgânicos. Essa classificação não sofreu uma revisão estrutural desde a sua concepção e, embora com uma grande utilidade potencial, contém problemas que limitam a possibilidade de seu uso para a gerência. Alguns desses problemas foram apontados no trabalho de Silver et al. 22: a classificação leva em consideração principalmente o procedimento realizado e, em poucos casos, acoplado ao código, há diferenciação entre criança e adulto ou se a cirurgia é ou não para neoplasma. Problemas sérios resultam da não consideração de outras variáveis que diferenciem os pacientes quanto à sua gravidade clínica e quanto ao perfil de consumo de recursos como, por exemplo: o diagnóstico principal, diagnósticos secundários, idade etc. Essa falha na classificação faz com que pacientes com diagnósticos diversos e gravidades distintas sejam classificados em um mesmo grupo, formando grupos de pacientes que têm certa homogeneidade quanto aos procedimentos realizados em sua estada hospitalar, mas que são clinicamente heterogêneos em termos da gravidade da doença ou de consumo de recursos, dificultando a realização de comparações. Outros problemas apontados pelos mesmos autores estão mais relacionados à lista de procedimentos: (1) a lista de procedimentos não contempla todas as possibilidades, por exemplo, os procedimentos clínicos, que são semelhantes aos diag- 
nósticos da Classificação Internacional de Doenças (CID-9), não englobam todas as opções de diagnóstico da mesma; (2) alguns procedimentos cirúrgicos não diferenciam os diferentes portes de cirurgia.

Embora no resumo de alta hospitalar (denominado AIH) haja informações sobre sexo, idade, diagnóstico, e na base de dados do SIH/ SUS contenha as características dos hospitais, essas informações não são utilizadas para a classificação, mas sim, repetidas na descrição ou no código de alguns procedimentos. Isso distorce a caracterização do procedimento, aumenta desnecessariamente o número destes e contribui para uma menor confiabilidade na anotação de informações importantes nos resumos de alta, como pode ser visto no estudo de Veras \& Martins 23. Em resumo, criou-se um sistema de informação abrangendo uma grande proporção das hospitalizações brasileiras, que permite seu uso além de propósitos financeiros. Esse sistema de informação trouxe embutido um sistema de classificação avançado para o momento em que foi criado, mas por falta de revisão e da incorporação dos avanços tecnológicos, tal classificação encontra-se hoje ultrapassada frente a outras desenvolvidas e aprimoradas ao longo do tempo.

Este trabalho centra-se na descrição da classificação e na análise dos potenciais usos dos AP-DRGs para discriminar o perfil de assistência de unidades hospitalares da região de $\mathrm{Ri}$ beirão Preto. As análises aqui apresentadas focalizam: (1) a comparação do tempo de permanência hospitalar por DRGs na região de Ribeirão Preto e Estados Unidos; e (2) o case-mix observado nos hospitais da região de Ribeirão Preto, destacando as diferenças entre eles e as relacionadas à procedência dos pacientes.

\section{Uma breve descrição da classificação AP-DRG}

Para se chegar ao AP-DRG, passa-se por uma árvore de decisões onde o primeiro passo é a classificação do diagnóstico principal em uma das 25 grandes categorias diagnósticas (GCD). Essas GCDs são semelhantes aos capítulos da CID, só que em maior número, principalmente devido à subdivisão de alguns capítulos da CID, como por exemplo: GCD 3 - doenças e desordens dos ouvidos, nariz, boca e garganta e GCD 20 - uso de álcool/drogas e doenças mentais orgânicas induzidas por álcool/drogas, e ao acréscimo de outros como a GCD 25 - trauma múltiplo, GCD 24 - Síndrome de Imunodeficiência Adquirida e a GCD 22 - queimaduras.
Cada paciente pertence a somente uma GCD. O segundo passo é a subdivisão das hospitalizações em aquelas que passaram ou não por um processo cirúrgico, criando um agrupamento cirúrgico e outro clínico. Os procedimentos cirúrgicos foram definidos como aqueles que são comumente realizados em centro cirúrgico. $\mathrm{O}$ terceiro passo é a classificação dos pacientes cirúrgicos pelas cirurgias específicas, organizadas, em geral, pela hierarquia de porte cirúrgico em: cirurgia maior, cirurgia menor, outra cirurgia, cirurgia não relacionada ao diagnóstico principal. Os pacientes classificados em grupos clínicos são organizados em subgrupos que geralmente correspondem a classes de neoplasmas, de sintomas e de condições específicas relativas ao sistema orgânico envolvido, além de uma classe residual. Todos os subgrupos formados também obedecem a princípios de organização que podem ser pela anatomia, pela etiologia, pelo processo de tratamento, pela forma de lidar com o diagnóstico ou pelo procedimento. Para diversos casos, a classificação termina nessa etapa e o paciente é classificado em um AP-DRG; para outros, há uma última etapa. O quarto passo da classificação consiste na consideração de outras informações que podem ser: a idade, os diagnósticos secundários (também denominados comorbidades e/ou complicações - CC), o destino após a alta e informações sobre a realização de alguns procedimentos considerados não cirúrgicos 24 .

Há algumas situações que fogem à regra geral como, por exemplo: os neonatos que são separados em uma GCD própria a partir da idade, tendo o peso ao nascer como primeira subdivisão; os casos de HIV que também vão para uma GCD própria; os pacientes que passaram por transplante e por traqueostomia que vão direto para AP-DRGs específicos.

Os diagnósticos secundários caracterizados como "comorbidade e/ou complicação" (CC) são freqüentes na caracterização dos APDRGs por consistirem em uma informação importante da complexidade do paciente. Dos diversos diagnósticos secundários presentes no resumo de alta do paciente, somente os que não fazem parte do processo da doença reportada como diagnóstico principal e que representam adição de complexidade na atenção hospitalar, principalmente quanto ao consumo de recursos, são considerados CC para a classificação dos AP-DRGs. Os diagnósticos secundários são considerados importantes não só na classificação em AP-DRGs mas, também, em diversas classificações que buscam um ajuste de risco do paciente, utilizadas principalmente para a análise de óbitos e avaliação 
da qualidade da atenção ou dos serviços hospitalares 10,11,12,13,16,25.

\section{Materiais e métodos}

Foi utilizada a base de dados de 166.282 hospitalizações ocorridas na região administrativa do Estado de São Paulo, denominada Diretoria Regional de Saúde 18 (DIR 18) no ano de 1997. Dos 36 hospitais da região, três não reportaram hospitalizações no ano em estudo e três foram excluídos das análises por serem hospitais de longa permanência. Para este estudo foram utilizadas informações de nove hospitais do $\mathrm{Mu}$ nicípio de Ribeirão Preto e 21 dos demais municípios da região. Esses dados foram cedidos pelo Centro de Processamento de Dados Hospitalares $(\mathrm{CPDH})$, sob responsabilidade do Departamento de Medicina Social da Faculdade de Medicina de Ribeirão Preto, Universidade de São Paulo. A região de Ribeirão Preto é a única no país que dispõe, desde a década de 70, de uma base de dados hospitalares constituída de informações dos resumos de saída hospitalares. Além das informações comuns em resumos de saída, há também quatro possibilidades de anotação de diagnósticos secundários, afora o diagnóstico principal, e três possibilidades de anotação de procedimentos cirúrgicos. Essa base de dados não se destina à remuneração e contempla a totalidade de unidades hospitalares públicas, privadas e filantrópicas da DIR 18.

A classificação das hospitalizações em DRGs foi realizada por meio do programa computacional Grouper, versão 14.1, que contempla o sistema AP-DRG. O sistema envolve 641 AP-DRGs, e o programa computacional foi cedido para esta pesquisa pela empresa $3 M^{\circledR}$, que mantém reservados sobre ele todos os direitos de cópia. Ao longo deste trabalho, a versão APDRG da classificação utilizada será referida como DRG simplesmente. A metodologia de adequação dos dados de Ribeirão Preto, que possibilitou a sua classificação em AP-DRG, pode ser vista em Noronha 26.

\section{Comparação do tempo de permanência hospitalar por DRGs na região de Ribeirão Preto e nos Estados Unidos}

Para as análises comparativas do tempo de permanência hospitalar entre os DRGs de Ribeirão Preto e dos Estados Unidos, foi necessário excluir os casos que apresentavam um tempo de permanência bem diferenciado da média do DRG a que pertenciam, pois esses influencia- vam os resultados estatísticos. Foram excluídas todas as hospitalizações (2.753 hospitalizações) cujo tempo de permanência correspondia a pelo menos 2,5 desvios-padrão acima ou abaixo da média do logaritmo do tempo de permanência hospitalar do DRG. A base de dados resultante ficou composta de 163.517 hospitalizações e 579 AP-DRGs.

As informações sobre as hospitalizações americanas classificadas em DRGs foram obtidas por intermédio da equipe de pesquisadores da empresa $3 \mathrm{M} /$ Health Information Systems. Essas informações fazem parte da Amostra Nacional de Hospitalizações dos Estados Unidos (Nationwide Inpatient Sample - NIS), obtida pelo Health Cost and Utilization Project (HCUP), referentes ao ano de 1997, liberação no 6 . A base de dados utilizada, resultante de uma amostra estratificada, compreende cerca de $20 \%$ das hospitalizações em hospitais comunitários, pagas pelos seguros nacionais Medicare e Medicaid, seguros privados e não assegurados, e envolve aproximadamente mil hospitais, distribuídos em 22 estados americanos. Do total de 6.994.852 hospitalizações, foram excluídas aquelas com tempo de permanência menor que 1 dia ou maior ou igual a 150 dias, e aquelas cujo custo foi menor que US\$200 ou maior que US\$ 800 mil. Resultaram 6.993.676 hospitalizações. Para este estudo foram fornecidas as estatísticas relativas ao tempo de permanência hospitalar por AP-DRGs, resultantes da retirada dos casos discrepantes, seguindo os mesmos critérios já mencionados como utilizados para as hospitalizações de Ribeirão Preto. Essas estatísticas correspondem, portanto, à análise de 6.934.102 hospitalizações, ou 99,1\% da base de dados original. Contemplaram, para cada APDRG, a descrição, número de pacientes e média, valores máximo e mínimo, desvio padrão e coeficiente de variação da permanência hospitalar. Vale ressaltar que nos resumos de alta dos Estados Unidos há possibilidade de anotação de até oito diagnósticos secundários e até seis intervenções cirúrgicas 27.

Nas duas bases de dados foram excluídos os AP-DRGs com menos de trinta ocorrências, resultando em análises estatísticas de 395 APDRGs, contendo 161.445 hospitalizações de Ribeirão Preto e 6.228.356 hospitalizações dos Estados Unidos. Foram selecionados para apresentação neste artigo, os AP-DRGs para os quais o teste T de Student indicou diferença na média dos tempos de permanência hospitalar observada entre a região de Ribeirão Preto e os Estados Unidos. 
Case-mix de hospitais da região de Ribeirão Preto

A complexidade do case-mix hospitalar relacionada ao consumo de recursos tem sido utilizada como um dos critérios para a alocação de recursos financeiros a hospitais 28,29. Para tal, há necessidade de apuração ou estimativa dos custos de cada DRG com o intuito de se estabelecer o seu peso relativo. Como este estudo não objetivou apurar ou estimar custos por DRG, para as análises da complexidade do case-mix hospitalar foram utilizados os pesos relativos dos custos de cada DRG, desenvolvidos pela empresa $3 M^{\circledR}$ Health Information Systems, para o Estado de Nova York em 1997, e cedidos para este trabalho. Os pesos variam de 36,8920 para o AP-DRG de transplante de pulmão a 0,1416 para falso trabalho de parto, tornando possível fazer um ranking dos AP-DRGs quanto a consumo de recursos. O somatório da multiplicação do volume de pacientes atendidos em cada AP-DRG pelo seu peso relativo, resulta no case-mix global de cada hospital.

Case- mix global $=\Sigma$ (número de saídas do AP-DRG X peso relativo dos AP-DRGs)

Retirando-se o efeito do volume de pacientes, com a divisão do case-mix global pelo volume de pacientes, a média do case-mix do hospital é obtida como observa-se na fórmula abaixo:

$$
\begin{aligned}
& \text { Índice ou média } \\
& \text { do } \text { case } \text {-mix }
\end{aligned}=\frac{\text { Case- } \text { mix } \text { global }}{\text { número total de saídas }}
$$

As análises do case-mix de hospitais da região de Ribeirão Preto foram realizadas com toda a base de dados da DIR 18, excluindo 779 saídas classificadas em DRGs que contêm erros ou são inválidos. Foram consideradas, portanto, 165.503 hospitalizações. O perfil de complexidade dos hospitais do município e região de Ribeirão Preto foi considerado em relação ao volume de hospitalizações de cada hospital e em relação à procedência geográfica dos pacientes com hospitalização paga pelo SUS. A base de dados utilizada contém a informação da forma de pagamento de cada internação podendo esta ser: particular, SUS e planos ou seguros de saúde. No caso específico de um hospital público, as 9.223 hospitalizações classificadas erroneamente como de indigentes foram incorporadas à categoria SUS. A variável procedência do paciente informa o município, se este for do Estado de São Paulo, ou o estado brasileiro ou país de procedência. Três hospitalizações foram excluídas por não terem a in- formação sobre a procedência. Para as análises iniciais foram selecionados os municípios que mais tiveram pacientes atendidos no Município de Ribeirão Preto, com base nos resultados do ranking do case-mix global.

\section{Resultados e discussão}

Na Tabela 1, pode-se observar exemplos de diversos DRGs e o tempo médio de permanência hospitalar (TMP) em Ribeirão Preto e Estados Unidos. Foram selecionados alguns DRGs que apresentaram, em Ribeirão Preto, médias de permanência superiores e inferiores às observadas nos Estados Unidos, com o objetivo de levantar hipóteses sobre as diferenças.

O diagnóstico principal, procedimentos, faixas etárias, peso ao nascer, tipo de alta e diagnósticos secundários (CC) são algumas das variáveis presentes na descrição dos DRGs selecionados. Essas variáveis fazem parte da árvore de decisões da classificação que resulta nos DRGs.

Na parte superior da Tabela 1 estão reunidos DRGs cuja média de permanência foi mais elevada em Ribeirão Preto, e na inferior, DRGs cuja média de permanência foi mais elevada nos Estados Unidos. Observa-se que em diversos DRGs de Ribeirão Preto a média de permanência é aproximadamente o dobro da dos Estados Unidos, embora muitas vezes, apresentando um coeficiente de variação próximo do observado para os Estados Unidos. Pela grande diferença na média de permanência, chamam a atenção o transplante renal e o DRG 623 de neonatos. A diferença de média de permanência do transplante renal pode decorrer de uma maior padronização da atenção nos Estados Unidos que em Ribeirão Preto, ou também, da possibilidade dos transplantados nos Estados Unidos serem transferidos a outras instituições como as nursing homes para a continuidade do tratamento, opção inexistente em Ribeirão Preto. Uma outra hipótese é a das pessoas transplantadas em Ribeirão Preto se encontrarem em um estado geral mais grave que os pacientes dos Estados Unidos. Esse DRG reúne todos os Transplantes Renais sem subdivisão por comorbidades e complicações ou faixas etárias. Pode ser que em Ribeirão Preto essas subdivisões sejam necessárias, pois o coeficiente de variação é bem mais elevado que nos Estados Unidos, apontando grande variabilidade do tempo de permanência. Uma outra hipótese é a de que os hospitais que realizam esse procedimento tenham processos de atenção diferenciados como, por exemplo, internar o paciente 
DRGs selecionados com médias de permanência hospitalar estatisticamente diferentes na comparação dos dados de Ribeirão Preto, São Paulo, Brasil, (DIR 18) e Estados Unidos, 1997.

\begin{tabular}{|c|c|c|c|c|c|c|c|}
\hline \multirow[t]{2}{*}{ DRG } & \multirow[t]{2}{*}{ Descrição } & \multicolumn{3}{|c|}{ Ribeirão Preto } & \multicolumn{3}{|c|}{ Estados Unidos } \\
\hline & & Saídas & Média & CV (\%) & Saídas & Média & $\mathrm{CV}(\%)$ \\
\hline 773 & $\begin{array}{l}\text { Pneumonia simples e pleurisia, idade }<18 \text { anos, } \\
\text { sem comorbidade e/ou complicação }\end{array}$ & 4.713 & 5 & 60 & 22.910 & 3 & 54 \\
\hline 772 & $\begin{array}{l}\text { Pneumonia simples e pleurisia, idade }<18 \text { anos, } \\
\text { com comorbidade e/ou complicação }\end{array}$ & 700 & 7 & 79 & 11.061 & 3 & 62 \\
\hline 121 & $\begin{array}{l}\text { Desordens circulatórias com infarto agudo do miocárdio } \\
\text { e complicação cardiovascular, alta vivo }\end{array}$ & 40 & 9 & 67 & 48.128 & 6 & 69 \\
\hline 122 & $\begin{array}{l}\text { Desordens circulatórias com Desordens circulatórias com infarto } \\
\text { sem complicação cardiovascular, alta vivo }\end{array}$ & 429 & 8 & 72 & 38.298 & 4 & 64 \\
\hline 126 & Endocardite aguda e subaguda & 33 & 25 & 81 & 2.690 & 12 & 83 \\
\hline 133 & Aterosclerose, sem comorbidade e/ou complicação & 103 & 6 & 108 & 28.162 & 2 & 62 \\
\hline 546 & Bypass coronariano com comorbidade e/ou complicação maior & 30 & 20 & 62 & 16.623 & 12 & 60 \\
\hline 107 & Bypass coronariano sem cateterismo cardíaco & 370 & 12 & 55 & 24.393 & 6 & 37 \\
\hline 295 & Diabetis, idade $<36$ anos & 186 & 5 & 84 & 14.288 & 3 & 60 \\
\hline 302 & Transplante renal & 36 & 35 & 84 & 2.968 & 9 & 58 \\
\hline 337 & Prostatectomia transuretral, sem comorbidade e/ou complicação & 366 & 4 & 52 & 13.432 & 2 & 51 \\
\hline 623 & $\begin{array}{l}\text { Neonato } \mathrm{c} / \text { peso ao nascer }>2.499 \mathrm{~g} \text {, com cirurgia significante, } \\
\text { sem problemas múltiplos maiores }\end{array}$ & 103 & 34 & 140 & 1.156 & 8 & 108 \\
\hline 458 & Queimaduras não extensas com enxerto de pele & 70 & 26 & 61 & 2.297 & 12 & 84 \\
\hline 2 & Craniotomia para trauma, idade $>17$ anos & 129 & 5 & 94 & 2.539 & 8 & 77 \\
\hline 482 & Traqueostomia com desordens da boca, laringe ou faringe & 38 & 5 & 79 & 2.965 & 11 & 77 \\
\hline 483 & Traqueostomia outra do que para desordens da boca, laringe ou faringe & 88 & 23 & 75 & 16.536 & 38 & 66 \\
\hline 165 & $\begin{array}{l}\text { Apendicectomia com diagnóstico principal Complicado, } \\
\text { sem comorbidade e/ou complicação }\end{array}$ & 215 & 2 & 77 & 7.067 & 4 & 53 \\
\hline 371 & Cesariana, sem comorbidade e/ou complicação & 9.127 & 2 & 31 & 97.962 & 3 & 27 \\
\hline 650 & Cesariana de alto risco, com comorbidade e/ou complicação & 73 & 5 & 71 & 9.889 & 6 & 72 \\
\hline 651 & Cesariana de alto risco, sem comorbidade e/ou complicação & 1.454 & 2 & 48 & 12.213 & 4 & 50 \\
\hline 430 & Psicoses & 778 & 5 & 143 & 176.158 & 9 & 90 \\
\hline 705 & HIV com infecções maiores relacionadas, com tuberculose & 40 & 9 & 91 & 262 & 15 & 91 \\
\hline
\end{tabular}

Saídas = inclui altas, óbitos e transferências; $C V$ = coeficiente de variação do tempo de permanência hospitalar; Média= média de permanência hospitalar.

por um período maior antes ou após a cirurgia, contribuindo para uma maior variação.

A grande diferença das médias de permanência do DRG 623 de Neonatos pode estar expressando a presença de casos com baixo peso ao nascer nesse DRG, que não estão classificados adequadamente devido à ausência dessa variável no resumo de alta de Ribeirão Preto. Os neonatos são classificados em DRGs a partir de subdivisões do peso que este apresentou ao nascer, e a ausência dessa informação fez com que todos os neonatos de Ribeirão Preto fossem considerados como acima de $2.499 \mathrm{~g}$. Portanto, nesse DRG podem estar incluídos alguns recém-natos de baixo ou baixíssimo peso ao nascer, situação essa que contribui também para a diminuição do case-mix global daqueles hospi- tais que atenderam casos graves, com consumo de recursos elevado.

Em Ribeirão Preto, entre os dois DRGs de Pneumonia, o DRG 772 apresenta uma média de permanência superior ao DRG 773, o que é esperado, uma vez que ele reúne casos com diagnósticos secundários relevantes, ou seja, de crianças e adolescentes em estados clinicamente mais graves. Por outro lado, observa-se que, nos Estados Unidos, as médias de permanência desses dois DRGs são aproximadamente iguais, contrariando as expectativas, e os coeficientes de variação das médias de permanência são inferiores aos de Ribeirão Preto. Algumas hipóteses podem ser levantadas para essa ocorrência nos Estados Unidos. Como os DRGs foram construídos inicialmente utilizan- 
do o tempo de permanência como referência do consumo de recursos e, posteriormente, ajustada pelos custos hospitalares, esse ajuste pode ter relativizado as diferenças do tempo de permanência nos Estados Unidos. Portanto, o DRG 772 pode estar reunindo, nos Estados Unidos, pacientes diferenciados em termos de gravidade clínica e de consumo de recursos, mas com pouco impacto no tempo de permanência hospitalar. Uma outra hipótese é a de que os pacientes classificados no DRG 772 possam estar tendo alta precoce nos Estados Unidos.

No conjunto de DRGs de Ribeirão Preto, cuja média de permanência apresentou-se inferior à observada nos Estados Unidos, verificase que, em diversos DRGs, esta foi metade da observada nos Estados Unidos. Os três DRGs de cesarianas sobressaem tanto pela diferença com os Estados Unidos como pela diferença entre eles em Ribeirão Preto. A cesariana sem complicação apresentou a mesma média de permanência da cesariana de alto risco, que reúne casos com descolamento prematuro de placenta, pré-eclâmpsia, diabetes, prematuridade etc. Muitas vezes, esses casos de cesariana com complicação reúnem gestantes que devem necessitar de internação mais longa para observação ou controle. O que os DRGs evidenciam é que isso pode não estar acontecendo em Ribeirão Preto. Por outro lado, as gestantes do DRG 650, que reúne pacientes de alto risco e com comorbidade e/ou complicação, apresentaram uma estada média hospitalar mais prolongada que a dos outros dois DRGs. Vale ressaltar que o tempo médio de permanência estipulado pelo SUS para a cesariana e para a cesariana de alto risco é de três dias, isto é, as gestantes nos DRGs 371 e 651 estão ficando menos tempo internadas que o estipulado pelo SUS. O coeficiente de variação do tempo de permanência desses dois DRGs não é elevado, apontando para pouca variação na região. Outros DRGs chamam a atenção como o de craniotomia, apendicectomia, HIV e de psicoses, que apresentaram uma média de permanência muito inferior à verificada nos Estados Unidos, apontando para a necessidade de uma melhor averiguação do processo de atendimento a esses pacientes pois, uma hipótese plausível, é que estes podem estar tendo alta hospitalar precoce.

O tempo médio de permanência hospitalar tem sido utilizado para reportar, principalmente, a eficiência da atenção hospitalar e subsidiar avaliações relacionadas à qualidade do cuidado. Essa informação apresentada como um valor agregado da instituição como um todo, ou mesmo sendo apresentada por grupos de diagnósticos ou por procedimentos, acaba sendo uma informação de importância limitada, fornecendo poucos subsídios para que o gestor possa intervir. Essa limitação é dada porque a informação não leva em consideração o perfil dos pacientes atendidos, ou seja, é assumido que a proporção do case-mix é constante dentro do hospital e entre hospitais ao longo do tempo, e que este atende tipos de casos homogêneos e produz tratamento homogêneo 30 . Como não se está falando de pacientes que possuem perfis semelhantes, comparações internas ou entre hospitais ficam comprometidas. Em resumo, não há reconhecimento de que os hospitais possuem diferentes recursos, pacientes com distintos perfis de complexidade clínica e que é utilizado um conjunto de bens e serviços hospitalares diferenciados para tratar esses pacientes. Alguns estudos vão tentando agrupar os pacientes por algumas variáveis para realizar análises. Pode-se observar isso em estudos como o de Yazlle-Rocha 31, que analisando hospitalizações de Ribeirão Preto ocorridas no ano de 1972, encontrou variações no TMP entre diferentes faixas etárias, sendo maior nos menores de 1 ano de idade e acima de 45 anos. Favero 32, complementou essas informações quando verificou que o TMP apresentou um padrão de regularidade entre 1971 e 1974 para as diferentes faixas etárias. Este foi elevado para os menores de 1 ano, atingindo os menores valores entre 14 e 15 anos, elevando-se a partir da faixa de 45 a 65 e atingindo um valor máximo acima de 65 anos. O caminho dessas análises é a de maior controle de variáveis que influenciam o tempo de permanência hospitalar como visto no estudo de Favero ${ }^{32}$, que observou que pacientes portadores de mais de um diagnóstico têm uma demora média maior, independentemente da presença ou não de cirurgias.

A classificação DRG já agrupa os pacientes utilizando diversas variáveis que têm impacto no consumo de recursos hospitalares podendo, portanto, ser utilizada para identificar as possíveis razões ou levantar hipóteses sobre as diferenças entre as médias de permanência entre os dois países, como discutidas acima. Outras análises também podem ser realizadas como a comparação, ao longo dos anos, da média de permanência de DRGs dentro de um hospital ou entre vários hospitais. Pode se tomar como referência a média de permanência de DRGs da região ou do Município de Ribeirão Preto para comparar com a observada nos hospitais e verificar as causas responsáveis pelas diferenças observadas. Essas informações são de relevância para o gestor hospitalar que pode 
ver em que problemas de saúde os recursos hospitalares são mais utilizados.

Na Tabela 2, pode-se observar o total de hospitalizações, o case-mix global e a média do $c a$ se-mix. Observando o total de saídas, verificase que o Município de Ribeirão Preto é o que concentra o maior volume de hospitalizações da DIR 18, sobressaindo-se o hospital 1, responsável por um terço das saídas do município. Nos demais municípios da região, dos 21 hospitais, somente o hospital 25 apresentou mais do que 8 mil saídas no ano em estudo. Verifica-se que os hospitais do Município de Ribeirão Preto também apresentaram maiores case-mix globais e médias de complexidade que os demais integrantes da DIR 18. Vale ressaltar que desses 21 hospitais, somente três possuem mais de cem leitos gerais disponíveis, nove possuem de 50 a 90 leitos e 10 hospitais abaixo de 50 leitos.

Estes resultados são, de certa forma, esperados já que o Município de Ribeirão Preto é referência da DIR 18. Os hospitais 1, 5 e 9 do Município de Ribeirão Preto apresentaram os case-mix global e média de complexidade mais elevados de toda a DIR 18. Os DRGs de maior case-mix global desses hospitais foram: o DRG 430 (psicoses) no hospital 1, e o DRG 108 (outro procedimento cardiotoráxico sem diagnóstico de anomalia congênita) nos hospitais 5 e 9. O hospital de número 4, que atendeu ao segundo maior volume de saídas de toda a DIR 18, apresentou um baixo valor de case-mix global. Isso ocorreu porque esse hospital atendeu a pacientes de baixa complexidade, em sua maioria partos e recém-nascidos sem complicação - o DRG de maior case-mix global foi o DRG 371 (cesariana sem comorbidade e/ou complicação) - consistindo em exemplo do efeito do ajuste pela complexidade dos pacientes. Observa-se, portanto, que a diferença dos resultados comparativos entre o volume de saídas e o case-mix global deve-se à introdução, neste último, do ajuste da complexidade dos pacientes. O hospital 1 apresentou um casemix médio de 1,3, e o hospital 5 de 1,6. A razão para tal diferença é a alta proporção de pacientes de baixa complexidade atendidos no hospital 1 e uma proporção concentrada em DRGs complexos atendida no hospital 5. No que diz respeito aos demais municípios da região de Ribeirão Preto, verifica-se que os hospitais atenderam a pacientes pouco complexos.

Essas informações permitem uma visão global da região e do Município de Ribeirão Preto, fornecendo uma primeira avaliação sobre o que realizam os hospitais da DIR 18. O case-mix global pode ser utilizado como referência na
Tabela 2

Total de saídas, case-mix global e média do case-mix, segundo hospitais do município e região de Ribeirão Preto, São Paulo, Brasil, 1997.

\begin{tabular}{|c|c|c|c|}
\hline Hospital & Saídas* & Case-mix global & $\begin{array}{l}\text { Média do } \\
\text { case-mix }\end{array}$ \\
\hline 1 & 36.411 & 47.956 & 1,3 \\
\hline 2 & 9.464 & 9.357 & 1,0 \\
\hline 3 & 4.363 & 3.867 & 0,9 \\
\hline 4 & 11.404 & 6.956 & 0,6 \\
\hline 5 & 9.622 & 15.119 & 1,6 \\
\hline 6 & 6.204 & 7.480 & 1,2 \\
\hline 7 & 4.413 & 4.327 & 1,0 \\
\hline 8 & 5.608 & 6.115 & 1,1 \\
\hline 9 & 10.852 & 12.177 & 1,1 \\
\hline Município Ribeirão Preto & 98.341 & 113.354 & 1,2 \\
\hline 10 & 2.857 & 2.392 & 0,8 \\
\hline 11 & 5.075 & 3.955 & 0,8 \\
\hline 12 & 5.603 & 4.822 & 0,9 \\
\hline 13 & 1.516 & 1.151 & 0,7 \\
\hline 14 & 6.150 & 4.414 & 0,7 \\
\hline 15 & 3.659 & 2.597 & 0,7 \\
\hline 16 & 3.840 & 2.884 & 0,7 \\
\hline 17 & 3.593 & 2.667 & 0,7 \\
\hline 18 & 2.152 & 1.524 & 0,7 \\
\hline 19 & 4.519 & 3.956 & 0,9 \\
\hline 20 & 2.174 & 1.423 & 0,7 \\
\hline 21 & 4.214 & 3.088 & 0,7 \\
\hline 22 & 91 & 69 & 0,8 \\
\hline 23 & 1.514 & 1.101 & 0,7 \\
\hline 24 & 7.510 & 6.155 & 0,8 \\
\hline 25 & 8.848 & 6.964 & 0,8 \\
\hline 26 & 2.162 & 1.670 & 0,8 \\
\hline 27 & 1.337 & 884 & 0,7 \\
\hline 28 & 7 & 6 & 0,8 \\
\hline 29 & 327 & 223 & 0,7 \\
\hline 30 & 14 & 6 & 0,5 \\
\hline Região Ribeirão Preto & 67.162 & 51.951 & 0,8 \\
\hline DIR 18 & 165.503 & 165.305 & 1,0 \\
\hline
\end{tabular}

Exclui as hospitalizações classificadas nos DRGs 469 e 470.

* Saídas - incluem altas, transferências e óbitos.

alocação de recursos financeiros aos diferentes serviços, por considerar não apenas o volume, mas também o perfil de complexidade dos pacientes. Ele é, portanto, um instrumento passível de ser utilizado para fins de decisão quanto ao financiamento, na ordenação dos serviços, de acordo com sua maior ou menor complexidade e, portanto, com o maior ou menor consumo de recursos. Essas informações complementam a tabela anterior, permitindo uma vi- 
são do conjunto da atenção prestada e dos problemas de saúde que consomem mais recursos (assumindo que os pesos relativos dos DRGs se adequam à realidade em estudo). Elas podem ser utilizadas no planejamento da atenção, na investigação de capacidades físicas e de recursos humanos, tanto pelo Município de Ribeirão Preto como pelas instâncias responsáveis pelas regiões do Estado de São Paulo. A classificação DRG pode ser utilizada para analisar os óbitos hospitalares, mas os resultados devem ser interpretados com cautela, pois essa versão da classificação não tem um ajuste apropriado do risco de morrer. As análises que descrevem as hospitalizações baseadas apenas nas características individuais dos pacientes, tais como sexo e faixa etária, fornecem uma visão apenas parcial das hospitalizações, geralmente no nível de grandes grupos e de algumas doenças específicas. Os DRGs facilitam a diferenciação das hospitalizações, visto que agrupam às características do paciente outras relativas ao processo de tratamento e à gravidade do seu estado de saúde, possibilitando uma visão global da atenção.

O perfil dos pacientes pagos pelo SUS, segundo a sua procedência, permite uma visão geral do sistema de referência dos hospitais do Município de Ribeirão Preto e do perfil de complexidade dos pacientes vindos de outros municípios. Na Tabela 3, observa-se que os pacientes vindos de outros municípios apresentam uma média de complexidade mais elevada que os residentes no próprio Município de Ribeirão Preto, podendo-se observar esse padrão em todos os hospitais. A média de complexidade dos pacientes atendidos pelo hospital 5 foi a mais elevada tanto para pacientes procedentes de outros municípios da DIR 18, como os de outras localidades do país. É importante notar que os hospitais do Município de Ribeirão Preto são referência para municípios de outras regiões do Estado de São Paulo e de outros estados do país. Verifica-se que $38 \%$ das hospitalizações do SUS no Município de Ribeirão Preto são de pacientes moradores em outros municípios, sendo que $22 \%$ destes, moradores em outros municípios da DIR 18. Chamam a atenção os hospitais 1 e 5 que tiveram, aproximadamente, $40 \%$ de seu case-mix global constituído de pacientes procedentes de outros municípios (Figura 1).

Na Figura 1 observa-se, para cada hospital, a proporção do case-mix global segundo a procedência dos pacientes atendidos. Verifica-se que os pacientes procedentes da região de Ribeirão Preto e de outros municípios tiveram uma participação importante quanto aos recursos consumidos nos hospitais do Município de Ribeirão Preto. Para os hospitais 1 e 5, os pacientes procedentes de outros municípios representaram aproximadamente $50 \%$ do case-mix global destes hospitais. Isso significa que cerca de $50 \%$ dos gastos desses hospitais foram com o atendimento a moradores de outros municípios.

A Tabela 4 apresenta o volume e o case-mix global de pacientes financiados pelo SUS, atendidos nos hospitais do Município de Ribeirão Preto, mas moradores de outros municípios. Foram selecionados os municípios e estados que apresentaram o maior valor de casemix global, ou seja, aqueles que exportaram o conjunto de pacientes de maior complexidade para serem atendidos no Município de Ribei-

Local de procedência dos pacientes pagos pelo SUS (número, case-mix global e case-mix médio) segundo hospitais do Município de Ribeirão Preto, São Paulo, Brasil*.

\begin{tabular}{|c|c|c|c|c|c|c|c|c|c|c|c|}
\hline \multirow[t]{2}{*}{ Hosp. } & \multicolumn{3}{|c|}{ Município de Ribeirão Preto } & \multicolumn{3}{|c|}{$\begin{array}{l}\text { Municípios da Região } \\
\text { de Ribeirão Preto }\end{array}$} & \multicolumn{3}{|c|}{$\begin{array}{c}\text { Outros municípios de São Paulo, } \\
\text { Estados, Países }\end{array}$} & \multicolumn{2}{|c|}{ Total } \\
\hline & saídas & $\begin{array}{l}\text { Case-mix } \\
\text { global }\end{array}$ & $\begin{array}{l}\text { Case-mix } \\
\text { médio }\end{array}$ & saídas & $\begin{array}{c}\text { Case-mix } \\
\text { global }\end{array}$ & $\begin{array}{l}\text { Case-mix } \\
\text { médio }\end{array}$ & saídas & $\begin{array}{l}\text { Case-mix } \\
\text { global }\end{array}$ & $\begin{array}{l}\text { Case-mix } \\
\text { médio }\end{array}$ & saídas & $\begin{array}{c}\text { Case-mix } \\
\text { global }\end{array}$ \\
\hline 1 & 20.182 & 23.677 & 1,2 & 8.525 & 12.173 & 1,4 & 7.381 & 11.700 & 1,6 & 36.088 & 47.550 \\
\hline 2 & 5.647 & 4.707 & 0,8 & 953 & 1.006 & 1,1 & 378 & 789 & 2,1 & 6.978 & 6.501 \\
\hline 5 & 260 & 765 & 2,9 & 97 & 314 & 3,2 & 106 & 343 & 3,2 & 463 & 1.422 \\
\hline 8 & 1.896 & 1.987 & 1,1 & 750 & 918 & 1,2 & 417 & 671 & 1,6 & 3.063 & 3.575 \\
\hline 9 & 5.351 & 4.276 & 0,8 & 1.114 & 1.439 & 1,3 & 206 & 578 & 2,8 & 6.671 & 6.293 \\
\hline Total & 33.336 & 35.412 & 1,1 & 11.439 & 15.850 & 1,4 & 8.488 & 14.080 & 1,7 & 53.263 & 65.341 \\
\hline
\end{tabular}

* Exclui as hospitalizações classificadas nos DRGs 469 e 470, 1 caso no hospital 1 e 1 no hospital 9 sem informação da procedência. 
rão Preto. O primeiro conjunto de municípios apresentados na Tabela 4 reúne os que fazem parte da região administrativa de Ribeirão Preto. Observando os totais da Tabela 3 e da Tabela 4 , verifica-se que esses sete municípios selecionados representaram $54 \%$ do volume de saídas e $52 \%$ do case-mix global dos pacientes procedentes da região de Ribeirão Preto. Os municípios de Sertãozinho e Batatais foram os que exportaram pacientes de maior complexidade média e acima da média do conjunto de municípios da região. Com exceção do Município de Brodosqui, todos os demais têm disponível, pelo menos, um hospital no município (em sua maioria, hospitais filantrópicos). É possível que essa busca por atendimento hospitalar no $\mathrm{Mu}$ nicípio de Ribeirão Preto seja, em parte, devido à disponibilidade de tecnologia e, em parte, pela grande proximidade desses municípios com Ribeirão Preto pois, com exceção dos municípios de Barrinha e de Batatais, todos os demais fazem divisa com o Município de Ribeirão Preto.

Os hospitais do município de Ribeirão Preto também atenderam a pacientes de 238 municípios do Estado de São Paulo que não fazem parte da DIR 18, mas relativamente poucas internações corresponderam a esse caso. Os sete municípios selecionados na Tabela 4 representaram 33\% tanto do volume de saídas como do case-mix global dos pacientes procedentes dos Municípios do Estado de São Paulo. Nota-se que a média do case-mix das hospitalizações desses municípios já é mais elevada que as observadas nos municípios da região de Ribeirão Preto.

Os Estados de Minas Gerais, Goiás e Paraná representaram $6 \%$ do case-mix global dos pacientes do SUS atendidos no Município de Ribeirão Preto, $86 \%$ do volume de saídas e $87 \%$ do case-mix global dos pacientes com procedência de outros estados do Brasil.

Espera-se que esses pacientes atendidos no Município de Ribeirão Preto tenham uma complexidade elevada ou tenham necessitado de serviços específicos oferecidos no município, que justifique a busca de hospitalização. Para as três categorias de procedência apresentadas, a GCD 1 (doenças e desordens do sistema nervoso) e a GCD 5 (doenças e desordens do sistema circulatório) foram as que apresentaram maior peso em termos de consumo de recursos, considerando-se o case-mix global. Sobressaíram o DRG 1 (craniotomia exceto para trauma, idade > 17 anos) e o DRG 108 (outro procedimento cardiotoráxico sem diagnóstico principal de anomalia congênita), como os que mais contribuíram para o case-mix global de pacientes procedentes de municípios externos ao de Ribeirão Preto.
Figura 1

Distribuição da proporção do case-mix global de hospitalizações pagas pelo SUS, segundo a procedência dos pacientes, por hospitais do Município de Ribeirão Preto, 1997.

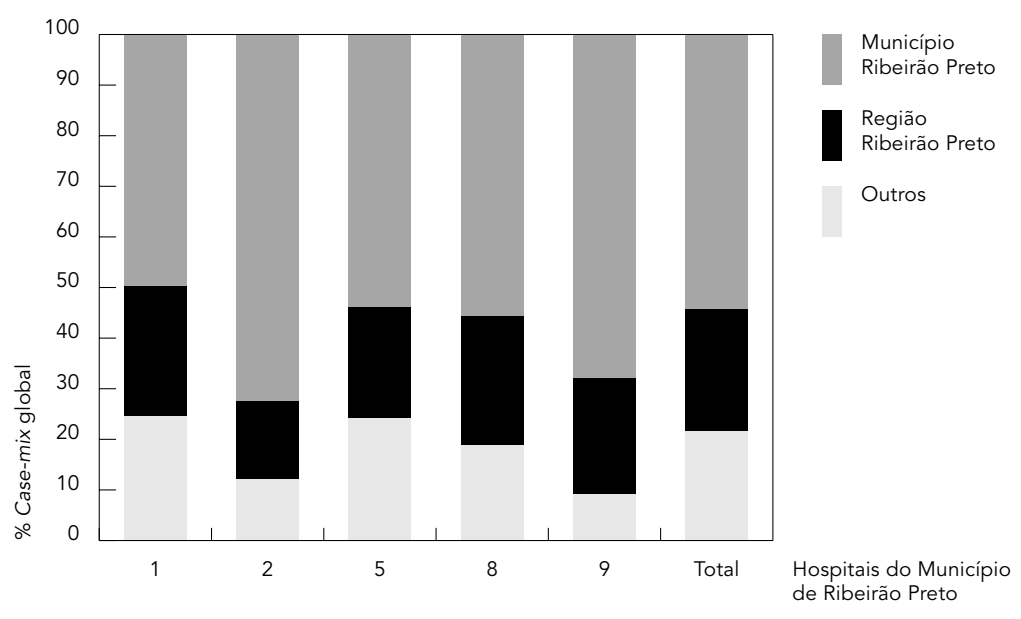

Com relação aos pacientes procedentes do próprio Município de Ribeirão Preto, as GCDs que apresentaram maior case-mix global foram: a GCD 5 (doenças e desordens do sistema circulatório) e a GCD 14 (gravidez, parto e puerpério). Os DRGs 127 (insuficiência cardíaca e choque) e 373 (parto vaginal sem menção de complicação) foram os que mais sobressaíram.

Para as autoridades municipais e gerentes hospitalares, as informações apresentadas são importantes para a implementação do SUS, principalmente para a reorganização regional da oferta de serviços e o financiamento dos cuidados à saúde. Vale ressaltar que o apresentado foi somente uma pequena amostra das informações que a classificação da base de dados de Ribeirão Preto em AP-DRGs pode viabilizar.

\section{Considerações finais}

Neste artigo, buscou-se descrever o sistema de classificação AP-DRG e apresentar algumas de suas potencialidades utilizando a base de dados da região de Ribeirão Preto. Nas análises aqui apresentadas, verificou-se que as diferenças no tempo médio de permanência entre Ribeirão Preto e os Estados Unidos podem estar sendo influenciadas por diferenças: na gerência desses pacientes, na disponibilidade de tecnologias, nas características dos hospitais, nas 
Tabela 4

Locais de procedência de pacientes atendidos em hospitais do Município de Ribeirão Preto, São Paulo, Brasil, pagos pelo SUS, selecionados pelo case-mix global mais elevado.

\begin{tabular}{|c|c|c|c|}
\hline Municípios/Estados & Saídas & $\begin{array}{l}\text { Case-mix } \\
\text { Global }\end{array}$ & $\begin{array}{l}\text { Case-mix } \\
\text { Média }\end{array}$ \\
\hline \multicolumn{4}{|c|}{$\begin{array}{l}\text { Municípios selecionados da Região } \\
\text { de Ribeirão Preto (DIR 18)* }\end{array}$} \\
\hline Barrinha & 1.055 & 1.215 & 1,2 \\
\hline Brodosqui & 972 & 1.025 & 1,1 \\
\hline Sertãozinho & 944 & 1.644 & 1,7 \\
\hline Serrana & 900 & 1.212 & 1,3 \\
\hline Jardinópolis & 859 & 1.029 & 1,2 \\
\hline Cravinhos & 792 & 1.011 & 1,3 \\
\hline Batatais & 612 & 1.062 & 1,7 \\
\hline Total & 6.134 & 8.200 & 1,3 \\
\hline \multicolumn{4}{|c|}{$\begin{array}{l}\text { Municípios selecionados do Estado } \\
\text { de São Paulo }\end{array}$} \\
\hline Franca & 482 & 736 & 1,5 \\
\hline São Joaquim da Barra & 318 & 613 & 1,9 \\
\hline Morro Agudo & 311 & 469 & 1,5 \\
\hline Ituverava & 233 & 363 & 1,6 \\
\hline Bebedouro & 228 & 346 & 1,5 \\
\hline São Carlos & 210 & 346 & 1,7 \\
\hline Porto Ferreira & 199 & 364 & 1,8 \\
\hline Total & 1.981 & 3.237 & 1,6 \\
\hline \multicolumn{4}{|l|}{ Estados selecionados } \\
\hline Minas Gerais & 2.037 & 3.399 & 1,7 \\
\hline Goiás & 83 & 164 & 2,0 \\
\hline Paraná & 46 & 91 & 2,0 \\
\hline Total & 2.166 & 3.654 & 1,7 \\
\hline
\end{tabular}

^Exclui o Município de Ribeirão Preto. dual e as Secretarias Municipais de Saúde com os diferentes tipos de prestadores, sobre o papel de cada unidade na rede e o perfil de pacientes a serem atendidos. Neste sentido, pode subsidiar a construção de um sistema regionalizado e hierarquizado de atenção à saúde, com uma definição mais clara das unidades de referência para os diversos tipos de atendimento. A identificação de serviços pouco utilizados e o planejamento de novos serviços, podem dar suporte a políticas de concentração ou redistribuição de serviços dentro de hospitais e, até mesmo, entre hospitais, para se obter economia de escala e melhor qualidade da atenção. No âmbito da gestão clínica, a classificação DRG permite analisar as relações existentes entre consumo de recursos, processo de tratamento e resultados alcançados. A definição do perfil de pacientes importados e exportados dos diferentes municípios, qualificando o fluxo de pacientes, permite o ressarcimento financeiro entre áreas geográficas.

Para a utilização do Sistema de Classificação DRG na região de Ribeirão Preto, avalia-se que alguns dos problemas relacionados às informações contidas na base de dados possam vir a ser sanados. A introdução da variável peso ao nascer ao resumo de saída se faz importante, assim como maior atenção ao registro de diagnósticos secundários, melhores critérios para escolha do diagnóstico principal e anotação completa dos diagnósticos pelos médicos. As informações do CPDH sobre os diagnósticos estão codificadas pela CID-10, e os procedimentos pela tradução da classificação em vigor nos Estados Unidos, facilitando a utilização da versão internacional, ou seja, os IR-DRGs. A utilização da base de dados, não só para análises epidemiológicas, mas também para a gerência dos hospitais da rede de atenção, pode vir a contribuir com a melhoria das informações.

Os governos Federal, Estadual e Municipal, têm utilizado a classificação de procedimentos para a remuneração dos hospitais que atendem pelo SUS. A classificação de pacientes AP-DRG ou sua versão revisada e mais atual (IR-DRG) são opções que mereceriam uma avaliação como alternativa ao sistema vigente. Mesmo que se optasse por pagar os hospitais que atendem pelo SUS por orçamento global, a classificação DRG seria uma referência importante para estabelecer o volume de recursos a serem pagos, constituindo uma base técnica de definição de parte do orçamento (baseado na produção ajustada pela complexidade), além de contribuir com informações para o monitoramento. Isto é, o repasse financeiro aos hospitais do SUS seria mais justo se fosse considerada a complexi- 
dade dos pacientes, e não simplesmente feito com base no procedimento realizado. A avaliação dos custos de cada DRG, adequando os pesos relativos à realidade brasileira, é uma etapa importante a ser realizada. Para tal, existe metodologia já aprimorada ao longo da existência da classificação DRG 33 .

É possível classificar as informações da base de dados do SIH-SUS em DRGs, embora algumas limitações possam ser vislumbradas: a tabela de procedimentos deverá passar por um mapeamento para os códigos utilizados nos Estados Unidos, onde é utilizada a classificação ICD-9-CM/Procedures (esse mapeamento já foi realizado para a tabela de procedimentos emitida em 1986 por Silver et al. 22). Como no resumo de alta hospitalar não há a informação nem do peso ao nascer do neonato, nem de seu diagnóstico, a classificação deste ficará preju- dicada (o campo de diagnósticos secundários da mãe, algumas vezes, é utilizado para diagnósticos de neonatos). Por ter somente um campo para o registro de diagnósticos secundários e um para os procedimentos, resultará na redução do número de pacientes classificados em DRGs que indiquem maior complexidade, como verificado por Zanetta 34 .

Por fim, avalia-se que a classificação fornece informações importantes para a região de Ribeirão Preto e para o SUS, tanto para: subsidiar decisões concernentes à organização da oferta de serviços hospitalares, instrumentalizar a gestão hospitalar nos âmbitos financeiro, administrativo e clínico, aprimorar o pagamento aos hospitais, como contribuir para se alcançar maior integralidade e eqüidade na gestão do sistema de saúde.

\section{Resumo}

Os All Patient Diagnosis Related Groups (AP-DRG) constituem-se em um sistema de classificação de pacientes internados em hospitais gerais, que reúne pacientes em grupos, considerando diversas características com impacto no consumo de recursos hospitalares e critérios clínicos. O AP-DRG tem suas diversas versões utilizadas para a gerência e como unidade de pagamento a hospitais. O objetivo deste artigo foi descrever a classificação, explorando suas potencialidades para gerar informações para a gestão de hospitais. Foi utilizada a base de dados da região de Ribeirão Preto, São Paulo Brasil, de 1997, que inclui unidades hospitalares públicas, privadas e filantrópicas. Foram realizadas comparações entre Ribeirão Preto e Estados Unidos quanto ao tempo médio de permanência hospitalar de AP-DRGs selecionados. Utilizando-se os pesos relacionados ao custo relativo dos DRGs, construídos para o Estado de Nova York, Estados Unidos, foi possível verificar o perfil da atenção prestada por trinta hospitais da região de Ribeirão Preto e também o perfil dos pacientes pagos pelo SUS procedentes de outras localidades. Avalia-se que a classificação fornece informações acerca do perfil de complexidade da atenção hospitalar, tanto no que diz respeito à regionalização e sua organização em níveis de complexidade, como para subsidiar as decisões de composição dessa rede de assistência, auxiliar no seu monitoramento e contribuir para o aprimoramento do pagamento aos hospitais.

Serviços de Saúde; Recursos em Saúde; Tempo de Permanência; Gestão de Qualidade

\section{Colaboradores}

M. F. Noronha foi a principal responsável pela elaboração do texto, que foi planejado e revisado junto a M. C. Portela e M. L. Lebrão.

\section{Agradecimentos}

Este trabalho é parte da tese de doutoramento da primeira autora, que recebeu bolsa de PICDT da CAPES e também obteve financiamento pelo Programa de Pesquisa Estratégica da Escola Nacional de Saúde Pública, Fundação Oswaldo Cruz. Os autores agradecem à Dra. Cristiani Vieira Machado, Dra. Joyce Mendes de Andrade Schramm, Marismary Horsth De Seta e Dra. Silvia Marta Porto pela leitura, comentários e preciosas sugestões. Agradecemos também à equipe do CPDH do Departamento de Medicina Social da Faculdade de Medicina de Ribeirão Preto, Universidade de São Paulo, e em especial ao Prof. Dr. Juan Stuardo Yazlle Rocha, pela disponibilização da base de dados e todo o apoio solicitado. À empresa $3 \mathrm{M}^{\circledR} / \mathrm{HIS}$, em especial, ao Dr. Russ Castioni, Dr. Jim Vertrees e Dr. Richard Freedman que viabilizaram o programa de classificação dos AP-DRGs, disponibilizaram as estatísticas americanas e deram todo o apoio solicitado. Aos pareceristas dos Cadernos de Saúde Pública, pelas sugestões de aprimoramento do artigo. 


\section{Referências}

1. Fetter RB. The DRG patient classification system Background. In: Fetter RB, Brand DA, Gamache D, editors. DRGs their design and development. Ann Arbor: Health Administration Press; 1991. p. 3-27.

2. Fetter RB, Freeman JL, Averill RF, Thompson JD. Case-mix definition by Diagnosis Related Groups. Med Care 1980; 18 Suppl:1-53.

3. Averill RF, Muldoon JH, Vertrees JC, Goldfield NI, Mullin RL, Fineram EC, et al. The evolution of casemix measurement using diagnosis related groups (DRGs). Working paper $3 M$ Health Information Systems. http://www.3mhis.com/us/health care/his/research_reports.jhtml (acessado em 30/ Jan/2004).

4. Mullin RL. Development of DRGs. In: Proceedings of International Conference on Management and Financing of Hospital Services. London: Health Systems Management Group da School of Organization and Management/Henry J. Kaiser Family Foundation; 1986. p. 15-8.

5. Fetter RB, Thompson JD. Inventors of DRG look at PPS now. Interview by Marybeth Burke. Hospitals 1992; 66:136-8.

6. Wiley MM. Hospital financing reform and casemix measurement: an international review. Health Care Financ Rev 1992; 13:119-33.

7. Averill RF, Goldfield N, Steinbeck B, Grant T, Muldoon J, Brough AJ, et al. Development of All Patient Refined DRGs (APR-DRGs). Working paper $3 M$ Health Information Systems. http://www. 3mhis.com/us/healthcare/his/research_reports.j html (acessado em 30/Jan/2004).

8. Mullin RL, Vertrees J, Freedman R, Castioni R, Tinker A. Case-mix analysis across patient populations and boundaries: A refined classification system designed specifically for international use. $3 \mathrm{M} /$ Health Information System, Connecticut. http://www.3mhis.com/us/healthcare/his/ research_reports.jhtml (acessado em 10/Mai/2004).

9. Mullin RL, Hughes J, Averill R. 2003. International Refined Diagnosis Related Groups version 2.0. In: Proceedings of the 19th International Case Mix Conference. Capitalizing on Case Mix: Gaining a Global Perspective. Washington DC: 3M Health Information Systems; 2003. p. 306-7.

10. Knaus WA, Wagner DP, Draper EA, Zimmerman JE, Bergner M, Bastos PG. The apache III: prognostic system - risk prediction of hospital mortality for critically ill hospitalised adults. Chest 1991; 100:1619-36.

11. Young WW, Swinkola RB, Zorn DM. The measurement of hospital case mix. Med Care 1982; 20:50112.

12. Horn SD, Horn RA. Reliability and validity of severity of illness index. Med Care 1986; 24:159-78.

13. Gonnella JS, Hornbrook MC, Louis DZ. Staging of disease: a case mix measurement. JAMA 1984; 251:637-44.

14. Brewster AC, Karlin BG, Hyde LA, Jacobs CM, Bradbury RC, Chae YM. MEDISGRPS: a clinically based approach to classifying hospital patient admission. Inquiry 1985; 22:377-87.

15. Fetter RB, Averill RF, Lichtenstein J, Freeman JL. Ambulatory visit groups: a framework for mea- suring productivity in ambulatory care. Health Serv Res 1984; 19:415-37.

16. Charlson ME, Pompei P, Ales KL, MacKenzie CR. A new method of classifying prognostic comorbidity in longitudinal studies: development and validation. J Chronic Dis 1987; 40:373-83.

17. Schneider KC, Lichtenstein JL, Freeman JL, Newbold RC, Fetter RB. The AVG system for ambulatory care. In: Fetter RB, Brand DA, Gamache D, editors. DRGs their design and development. Ann Arbor: Health Administration Press; 1991. p. 291306.

18. Brand DA, Smits HL, Cooney Jr. LM, Scheneider KC, Murtaugh CM, Fetter RB. The PDG system for long-term care. In: Fetter RB, Brand DA, Gamache D, editors. DRGs their design and development. Ann Arbor: Health Administration Press; 1991. p. 307-32.

19. Goldfield N, Averill RF, Grant T, Gregg LW. The clinical development of an ambulatory classification system: version 2.0 Ambulatory Patient Groups. J Ambul Care Manage 1997; 20:49-56.

20. Iezzoni LI. Getting started and defining terms. In: Iezzoni LI, editor. Risk adjustment for measuring health care outcomes. Chicago: Health Administration Press; 2003. p. 17-32.

21. Levcovitz E, Pereira TRC. SIH/SUS (Sistema AIH): uma análise do sistema público de remuneração de internações hospitalares no Brasil - 1983 1991. Rio de Janeiro: Instituto de Medicina Social, Universidade do Estado do Rio de Janeiro; 1993. (Série Estudos em Saúde Coletiva no 57).

22. Silver LD, Travassos VC, Noronha MF, Martins MS, Leite IC. Estudo da validade dos Diagnosis Related Groups (DRG) para internações hospitalares no Brasil. Rio de Janeiro: Escola Nacional de Saúde Pública, Fundação Oswaldo Cruz; 1992. (Relatório final de pesquisa).

23. Veras CMT, Martins MS. A confiabilidade dos dados nos formulários de autorização de internação hospitalar (AIH), Rio de Janeiro, Brasil. Cad Saúde Pública 1994; 10:339-55.

24. Averill RF. Design and development of the All Patient Diagnosis Related Groups (AP-DRGs). In: 3M Health Information Systems, editor. All Patient Diagnosis Related Groups (AP-DRGs), version 14.0 definitions manual. Wallingford: New York State Department of Health/3M Health Information Systems; 1996.

25. Iezzoni LI. Range of risk factors. In: Iezzoni LI, editor. Risk adjustment for measuring health care outcomes. Chicago: Health Administration Press; 2003. p. 33-70.

26. Noronha MF. Classificação de hospitalizações em Ribeirão Preto: os Diagnosis Related Groups [Tese de Doutorado]. São Paulo: Faculdade de Saúde Pública de São Paulo, Universidade de São Paulo; 2001.

27. Iezzoni LI. Coded data from administrative sources. In: Iezzoni LI, editor. Risk adjustment for measuring health care outcomes. Chicago: Health Administration Press; 2003. p. 83-138.

28. Urbano J. Implementation of Diagnosis Related Groups in Portugal. In: Proceedings of Interna- 
tional Conference on Management and Financing of Hospital Services; London: Health Systems Management Group da School of Organization and Management/Henry J. Kaiser Family Foundation; 1986. p. 93-8.

29. Urbano J, Bentes M, Vertrees JC. Portugal: National Commitment and the implementation of DRGs. In: Kimberly JR, Pouvourville G, editors. The migration of managerial innovation: Diagnosis related groups and health care administration in Western Europe. San Francisco: Jossey-Bass Publishers; 1993. p. 215-53.

30. Hornbrook MC. Hospital case mix: its definition, measurement and use: Part I. The conceptual framework. Med Care Rev 1982; 39:1-72.

31. Yazlle-Rocha JS. Utilização dos leitos hospitalares gerais em Ribeirão Preto, São Paulo (Brasil). Rev Saúde Pública 1975; 9:477-93.

32. Favero M. Estudo da duração da internação em hospitais gerais de Ribeirão Preto, São Paulo [Tese Livre Docência]. Ribeirão Preto: Faculdade de Medicina de Ribeirão Preto, Universidade de São Paulo; 1975.

33. Chandler IR, Fetter RB, Newbold RC. Cost accounting and budgeting. In: Fetter RB, Brand DA, Gamache D, editors. DRGs their design and development. Ann Arbor: Health Administration Press; 1991. p. 91-120.

34. Zanetta SFR. Morbidade no Hospital das Clínicas: identificação de perfis e desenvolvimento de instrumento de monitoramento [Dissertação de Mestrado]. São Paulo: Faculdade de Medicina, Universidade de São Paulo; 2003.

Recebido em 26/Fev/2004

Versão final reapresentada em 25/Jun/2004

Aprovado em 02/Jul/2004 